\title{
Cycle Embedding in Generalized Recursive Circulant Graphs
}

\author{
Shyue-Ming TANG ${ }^{\dagger \mathrm{a})}$, Yue-Li WANG ${ }^{\dagger \dagger b)}$, Chien-Yi LI ${ }^{\dagger \dagger}$, Nonmembers, and Jou-Ming CHANG ${ }^{\dagger \dagger \dagger c)}$, Member
}

SUMMARY Generalized recursive circulant graphs (GRCGs for short) are a generalization of recursive circulant graphs and provide a new type of topology for interconnection networks. A graph of $n$ vertices is said to be $s$-pancyclic for some $3 \leqslant s \leqslant n$ if it contains cycles of every length $t$ for $s \leqslant t \leqslant n$. The pancyclicity of recursive circulant graphs was investigated by Araki and Shibata (Inf. Process. Lett. vol.81, no.4, pp.187-190, 2002). In this paper, we are concerned with the $s$-pancyclicity of GRCGs.

key words: interconnection networks, generalized recursive circulant graphs, recursive circulant graphs, cycle embedding, pancyclicity, bipancyclicity

\section{Introduction}

Interconnection networks are usually modeled as undirected simple graphs $G=(V, E)$, where the vertex set $V(=V(G))$ and the edge set $E(=E(G))$ represent the set of processors and the set of communication channels between processors, respectively. For graph embedding, it has many practical applications such as allocating concurrent processes to processors in networks, transplanting or simulating parallel algorithms developed for one network to a different one, and so forth. In particular, cycle embedding is an extension of the theoretical research on Hamiltonicity in graph theory. A ring structure is often used as a interconnection architecture for local area network and as a control and data flow structure in parallel and distributed networks. Many efficient algorithms with low communication cost have been developed based on the ring structure. Accordingly, cycle embedding is an important measurement in evaluating the efficiency of interconnection networks (see [13], [28], [29] and quotes therein).

Adding chords to a ring is to enhance its fault-tolerance capability. A circulant graph $C\left(n ; c_{1}, c_{2}, \ldots, c_{k}\right)$ is a ring of $n(\geqslant 3)$ vertices in which vertices $u$ and $v$ are adjacent if and only if $u \equiv v \pm c_{i}(\bmod n)$ where $u, v \in\{0,1, \ldots, n-1\}$ and $1 \leqslant$

Manuscript received January 4, 2018.

Manuscript revised March 27, 2018.

Manuscript publicized September 18, 2018.

${ }^{\dagger}$ The author is with the Department of Psychology and Social Work, National Defense University, Taipei, Taiwan, ROC.

${ }^{\dagger \dagger}$ The authors are with the Department of Information Management, National Taiwan University of Science and Technology, Taipei, Taiwan, ROC.

${ }^{\dagger \dagger}$ The author is with the Institute of Information and Decision Sciences, National Taipei University of Business, Taipei, Taiwan, ROC.

a) E-mail: tang1119@gmail.com

b) E-mail: ylwang@cs.ntust.edu.tw

c) E-mail: spade@ntub.edu.tw (Corresponding author)

DOI: $10.1587 /$ transinf.2018PAP0009 $c_{i}<c_{i+1} \leqslant\lfloor n / 2\rfloor$ for $1 \leqslant i \leqslant k-1$ (see [7] and [8, pp. 73$75])$. For example, two circulant graphs $C(24 ; 1,3,12)$ and $C(24 ; 1,4,8)$ are shown in Figs. 1 (a) and 1(b), respectively. In this figure, vertices are labeled by serial numbers within a circle. Since the two graphs belong to a class of generalized recursive circulant graphs (defined later in Sect.2), there is another labeling of vertices to simplify the structure representation. Circulant graphs, which are vertex-symmetric, form a subclass of Cayley graphs [1], [4].

A subclass of circulant graphs with recursive struc-

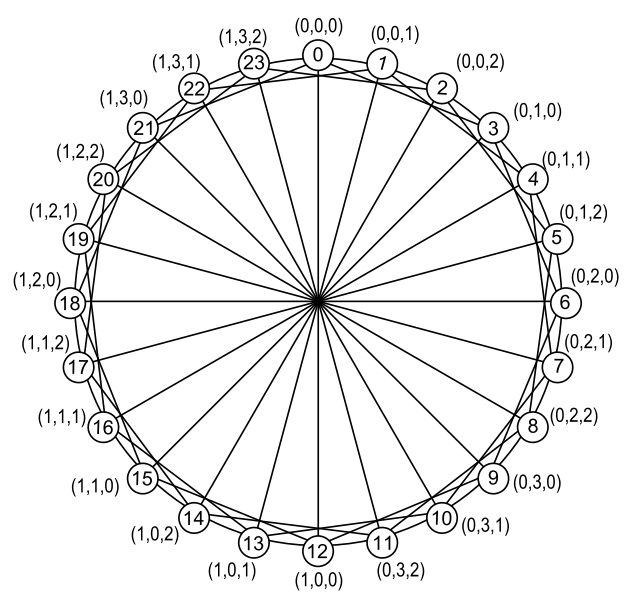

(a)

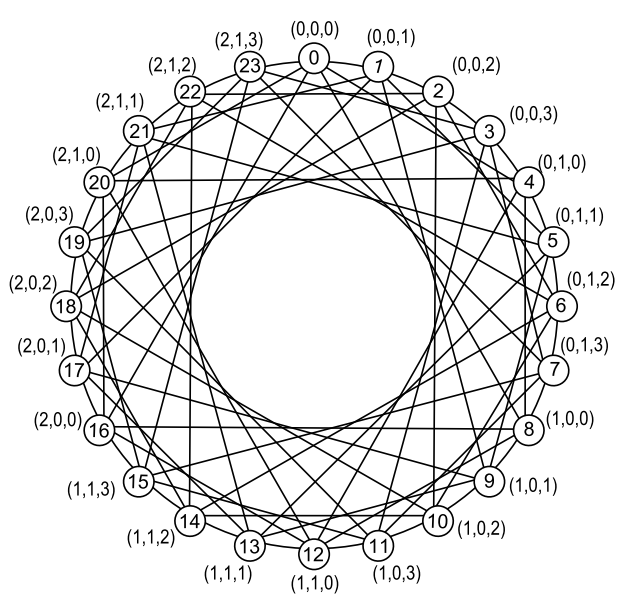

(b)

Fig. 1 Two generalized recursive circulant graphs: (a) $\operatorname{GR}(2,4,3)$ and (b) $\operatorname{GR}(3,2,4)$ 
ture was proposed by Park and Chwa [22] in the earlier stage. A circulant graph is called a recursive circulant graph (RCG for short), denoted by $R\left(c d^{m}, d\right)$, if $n=c \cdot d^{m}$ with positive integers $c<d$ such that $c_{i}=d^{i-1}$ for $i=1,2, \ldots, k$ and $k=\left\lceil\log _{d} n\right\rceil$. Note that if $c>1$, then $k=m+1$; otherwise, $k=m$. An RCG can also be constructed by $d$ disjoint copies of $R\left(c d^{m-1}, d\right)$ recursively, this is the reason why the name of these graphs contains the word "recursive". In $R\left(c d^{m}, d\right)$, since $c_{1}=$ $d^{0}=1$, all edges of $(u, u+1(\bmod n))$ form a Hamiltonian cycle and we call this Hamiltonian cycle the basic cycle. As a famous network topology, properties and algorithms on RCGs have been widely studied, such as Hamiltonian decomposition [5], [20], super-connectivity [27], faulttolerant Hamiltonicity [10], [21], [26], independent spanning trees [30], [31], disjoint path covers [16], [17], and routine and broadcasting schemes [12], [15], [23], [24]. Moreover, embedding schemes on RCGs are of particularly interested for many researches, e.g., path and cycle embeddings [2], [3], [21], tree embeddings [14], [18], and hypercube and meshe embeddings [23].

Although the class of RCGs can provide a good flexibility on designing network systems, due to the restriction of $0<c<d$ we can find that $C(12 ; 1,4)$ is an RCG (i.e., it is isomorphic with $R\left(3 \cdot 4^{1}, 4\right)$ ), while $C(12 ; 1,3)$ is not. To remove this restriction, Tang et al. [25] proposed a more generalized definition of RCGs, called generalized recursive circulant graphs (defined later in Sect. 2), which can also be constructed recursively.

To study cycle embedding, we need the following graph terminology. A graph of $n$ vertices is said to be $s$ pancyclic for some $3 \leqslant s \leqslant n$ if it contains cycles of every length $t$ for $s \leqslant t \leqslant n$. If $s=3$, then $s$-pancyclic is the so-called pancyclic [3], [21], [29]. A graph is called bipancyclic if it has cycles of every even length.

\section{Generalized Recursive Circulant Graphs}

A $k$-dimensional generalized recursive circulant graph ( $k$ GRCG for short) is denoted by $\operatorname{GR}\left(h_{k}, h_{k-1}, \ldots, h_{1}\right)$, where $h_{i} \geqslant 2$ is the size of the $i$ th dimension for $1 \leqslant i \leqslant k$. Every vertex $x$ in the graph is labeled by a $k$-tuple $\left(x_{k}, x_{k-1}, \ldots, x_{1}\right)$ with $0 \leqslant x_{i} \leqslant h_{i}-1$ for $1 \leqslant i \leqslant k$, which is a mixed radix number representation, such that it is adjacent to vertices $\left(x_{k}, x_{k-1}, \ldots, x_{i}+1, \ldots, x_{1}\right)$ and $\left(x_{k}, x_{k-1}, \ldots, x_{i}-1, \ldots, x_{1}\right)$, where the addition and subtraction in each dimension are with the carry and borrow. That is, for $x=\left(x_{k}, x_{k-1}, \ldots, x_{i}+\right.$ $\left.1, \ldots, x_{1}\right)$, if $x_{i}+1=h_{i}$, then a carry occurs at dimension $i$ and $x$ is indeed $\left(x_{k}, x_{k-1}, \ldots, x_{i+1}+1,0, x_{i-1}, \ldots, x_{1}\right)$. Similarly, for $x=\left(x_{k}, x_{k-1}, \ldots, x_{i}-1, \ldots, x_{1}\right)$, if $x_{i}=0$, then $x_{i}-1$ will borrow 1 from $x_{i+1}$, and the resulting label will be $\left(x_{k}, x_{k-1}, \ldots, x_{i+1}-1, h_{i}-1, x_{i-1}, \ldots, x_{1}\right)$. Furthermore, when the carry and borrow operations occur at dimension $k$, it is manipulated as that there is an invisible dimension $k+1$ in which no carry and borrow will occur. This means that vertices $\left(0, x_{k-1}, \ldots, x_{i}, \ldots, x_{1}\right)$ and $\left(h_{k}-1, x_{k-1}, \ldots, x_{i}, \ldots, x_{1}\right)$ are adjacent.

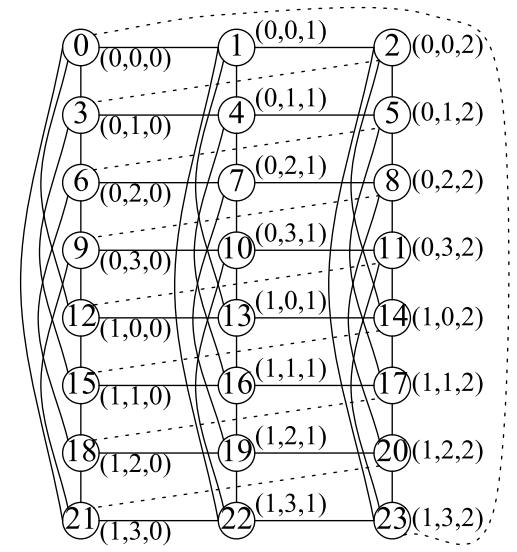

Fig. 2 The embedding of an $8 \times 3$ mesh on $\operatorname{GR}(2,4,3)$.

Clearly, $\operatorname{GR}\left(h_{k}, h_{k-1}, \ldots, h_{1}\right)$ contains $\prod_{i=1}^{k} h_{i}$ vertices, and in general, it is $2 k$-regular except for $(2 k-1)$-regular when $h_{k}=2\left(\right.$ since $x_{k}+1 \equiv x_{k}-1\left(\bmod h_{k}\right)$ in this case $)$. In [25], Tang et al. gave a mapping from $\operatorname{GR}\left(h_{k}, h_{k-1}, \ldots, h_{1}\right)$ to $C\left(n ; c_{1}, c_{2}, \ldots, c_{k}\right)$ which is described as follows.

Theorem 1 ([25]): A circulant graph $C\left(n ; c_{1}, c_{2}, \ldots, c_{k}\right)$ is $\operatorname{GR}\left(h_{k}, h_{k-1}, \ldots, h_{1}\right)$ if and only if $c_{i}=n / \prod_{j=i}^{k} h_{j}$ for $i=$ $1,2, \ldots, k$.

By Theorem 1, we can find that $C(24 ; 1,3,12)$ and $C(24 ; 1,4,8)$ shown in Figs. 1 (a) and 1 (b), respectively, are $\operatorname{GR}(2,4,3)$ and $\operatorname{GR}(3,2,4)$.

The following lemma provides a simple rule to determine whether a $k$-GRCG is bipartite or not.

Lemma 2 ([25]): $\operatorname{GR}\left(h_{k}, h_{k-1}, \ldots, h_{1}\right)$ is a bipartite graph if and only if $h_{k}$ is even and $h_{i}$ is odd for $i=1,2, \ldots, k-1$.

A mesh network is defined as the Cartesian product $P_{r} \times P_{s}$ of undirected paths $P_{r}$ and $P_{s}$, denoted by $M_{r \times s}$. Lemma 3 gives a condition that a mesh can be embedded in a $k$-GRCG. For example, we consider mesh embedding in $\operatorname{GR}(2,4,3)$. According to this lemma, all subgraphs of mech $M_{2 \times 12}$ or $M_{8 \times 3}$ can be embedded in $\operatorname{GR}(2,4,3)$. See Fig. 2 as an example.

Lemma 3 ([25]): For a mesh $M_{r \times s}$ and a $k$-GRCG $\operatorname{GR}\left(h_{k}, h_{k-1}, \ldots, h_{1}\right)$, if there exists an integer $j$ with $1 \leqslant$ $j \leqslant k$ such that $2 \leqslant r \leqslant \prod_{i=1}^{j} h_{i}$ and $2 \leqslant s \leqslant \prod_{i=j+1}^{k} h_{i}$, then $M_{r \times s}$ can be embedded in GR $\left(h_{k}, h_{k-1}, \ldots, h_{1}\right)$.

For more results related to $k$-GRCGs, we refer to [9], [11].

\section{The Pancyclicity of $k$-GRCGs}

In [3], Araki and Shibata addressed the pancyclicity on RCGs. In this section, we are concerned with the pancyclicity of $k$-GRCGs. Since the class of RCGs is a subclass of $k$-GRCGs, our results also hold for RGCs. We first examine the bipancyclicity of $k$-GRCGs. Clearly, a 1 -GRCG is a cycle and is not pancyclic except GR(3). Thus, we consider hereafter only $k$-GRCGs with $k \geqslant 2$. For simplicity, 
hereafter $\operatorname{GR}\left(h_{k}, h_{k-1}, \ldots, h_{1}\right)$ is abbreviated as GR when the context is clear.

By Lemma 3, we know that a mesh $M_{r \times s}$ with $r, s \geqslant 2$ and $r \cdot s=\prod_{i=1}^{k} h_{i}$ can be embedded in a $k$-GRCG. It has been proved that a mesh $M_{r \times s}$ is bipancyclic if both $r, s \geqslant 2$ (see [19, Lemma 2.1]). Since, by definition, the size of each dimension in a $k$-GRCG is at least 2 , the following result directly holds.

Lemma 4: For $k \geqslant 2$, any $k$-GRCG with $n(\geqslant 4)$ vertices is bipancyclic.

In the following, we shall investigate the existence conditions of odd cycles in $k$-GRCGs. For ease of description, we define some terms for using later. Hereafter, we assume that there are $n$ vertices in a $k$-GRCG, i.e., $n=\prod_{i=1}^{k} h_{i}$. A cycle of $t$ vertices is called a $t$-cycle and is denoted by $C_{t}$. If $t$ is even, then $C_{t}$ is called an even cycle; otherwise, an odd cycle. Obviously, for a $t$-cycle and a $w$-cycle, if there is exactly one common edge between them, then removing the common edge results in a $(t+w-2)$-cycle., denoted by $C_{t} \circ C_{w}$. We say that vertex $x=\left(x_{k}, x_{k-1}, \ldots, x_{i}, \ldots, x_{1}\right)$ takes jump $i^{+}\left(\right.$resp., $\left.i^{-}\right)$to reach vertex $y=\left(x_{k}, x_{k-1}, \ldots, x_{i}+1, \ldots, x_{1}\right)$ (resp., $\left.y=\left(x_{k}, x_{k-1}, \ldots, x_{i}-1, \ldots, x_{1}\right)\right)$ and denote by $x \stackrel{i^{+}}{\rightarrow} y$ (resp., $x \stackrel{i-}{\rightarrow} y$ ). In case of $h_{k}=2$, jumps $k^{+}$and $k^{-}$reach the same vertex and thus are viewed as one single jump $k^{-}$. When a jump $i^{p}$ where $p \in\{+,-\}$ is used consecutively $j$ times from vertex $x$ to vertex $y$, we use $x \stackrel{i^{p j}}{\rightarrow} y$ to denote it. Let $\delta$ stand for the minimum odd number, if it exists, in the set $\left\{h_{k}, h_{k-1}+1, h_{k-2}+1, \ldots, h_{1}+1\right\}$. A cycle $C_{t}$ passing through vertices $x_{1}, x_{2}, \ldots, x_{t}$ is denoted by $\left(x_{1}, x_{2}, \ldots, x_{t}, x_{1}\right)$ or in the simplified jump form $x_{1} \stackrel{j_{1} j_{2} \cdots j_{t}}{\longrightarrow} x_{1}$ where $j_{i}$ is the jump between $x_{i}$ and $x_{i+1}$.

For example, we consider $\operatorname{GR}(2,4,3)$ in Fig. 2. Vertex $(1,3,0)$ reaches vertices $(0,3,0),(0,0,0),(1,2,0),(1,3,1)$ and $(1,2,2)$ by taking jumps $3^{-}, 2^{+}, 2^{-}, 1^{+}$, and $1^{-}$, respectively. The 4 -cycle $((0,0,0),(0,0,1),(0,0,2),(0,1,0)$, $(0,0,0))$ can be represented as $(0,0,0) \stackrel{1^{+3}}{\rightarrow}(0,1,0) \stackrel{2^{-}}{\rightarrow}(0,0,0)$ or $(0,0,0) \stackrel{1^{+3} 2^{-}}{\longrightarrow}(0,0,0)$. The minimum odd number $\delta$ in the set $\left\{h_{3}, h_{2}+1, h_{1}+1\right\}=\{2,4+1,3+1\}$ is 5 .

Proposition 5: For any two jumps $j_{1}$ and $j_{2}$, if $x \stackrel{j_{1} j_{2}}{\longrightarrow} y$, then $x \stackrel{j_{2} j_{1}}{\longrightarrow} y$.

Lemma 6: For $\operatorname{GR}\left(h_{k}, h_{k-1}, \ldots, h_{1}\right)$, there exist cycles of lengths $h_{i}+1$ for $i=1,2, \ldots, k-1$. Furthermore, there is a cycle of length $h_{k}$ if $h_{k} \geqslant 3$.

Proof. Let $x=\left(x_{k}, x_{k-1}, \ldots, x_{i}, \ldots, x_{1}\right)$ be any vertex in GR. If we can prove that no vertex appears more than once in the path $x \stackrel{i^{+h_{i}}}{\rightarrow} y \stackrel{(i+1)^{-}}{\longrightarrow} z$ for $i=1,2, \ldots, k-1$ except that $x=z$, then the existence of $\left(h_{i}+1\right)$-cycle holds. By definition, all vertices in the path $x \stackrel{i^{+h_{i}} \rightarrow}{\rightarrow} y$ are distinct and, further, $y=$ $\left(x_{k}, x_{k-1}, \ldots, x_{i+1}+1, x_{i}, \ldots, x_{1}\right)$. Thus, after $y$ taking jump $(i+1)^{-}$, the label of $z$ will be $\left(x_{k}, x_{k-1}, \ldots, x_{i+1}, x_{i}, \ldots, x_{1}\right)$ which is exactly $x$. Therefore, there exist cycles of lengths

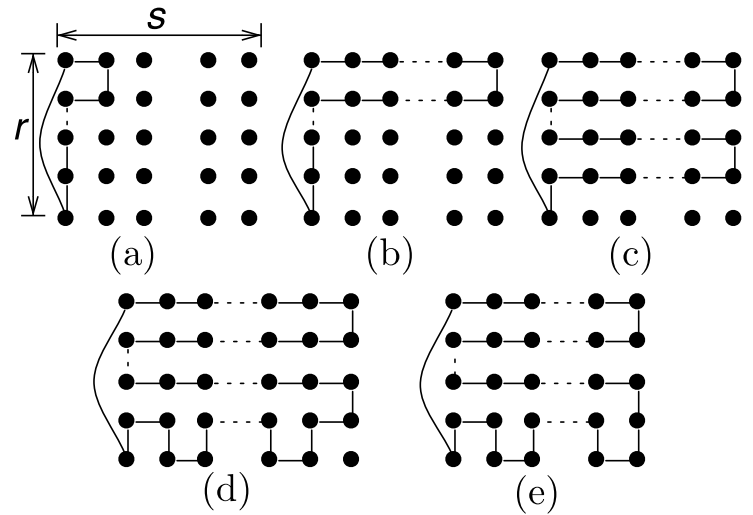

Fig. 3 Illustrations for Lemma 7.

$h_{i}+1$ for $i=1,2, \ldots, k-1$.

To prove the existence of $C_{h_{k}}$, it suffices to show that $x \stackrel{k^{+h h_{k}}}{\longrightarrow} z$ is an $h_{k}$-cycle. By using a similar argument as the previous case and the boundary property of dimension $h_{k}$, this case is also true. This completes the proof.

Lemma 7: If $\mathrm{GR}\left(h_{k}, h_{k-1}, \ldots, h_{1}\right)$ has $h_{k}=\delta$, then it contains odd cycles of every length in the range from $\delta$ to $n$.

Proof. By Lemma 3, a mesh $M_{r \times s}$ with $r=h_{k}$ and $s=\prod_{i=1}^{k-1} h_{i}$ can be embedded in GR. By definition, vertices in the first column of $M_{r \times s}$ form an $r$-cycle. Let $C_{r}=(0,0, \ldots, 0) \stackrel{k^{+h_{k}}}{\longrightarrow}(0,0, \ldots, 0)$ be the $r$-cycle in the first column, and let $C_{4}$ be the cycle $(0,0, \ldots, 0) \stackrel{1^{+} k^{+} 1^{-} k^{-}}{\longrightarrow}$ $(0,0, \ldots, 0)$ in $M_{r \times s}$. Then, $C_{r} \circ C_{4}$ results in a $C_{r+2}$ (see Fig. 3 (a)). Again, $C_{r+2} \circ C_{4}^{\prime}$ for the above $C_{r+2}$ and $C_{4}^{\prime}=$ $(0,0, \ldots, 1) \stackrel{1^{+} k^{+} 1^{-} k^{-}}{\longrightarrow}(0,0, \ldots, 1)$ will result in a $C_{r+4}$. By using the similar technique repeatedly on the first two rows of $M_{r \times s}$, we can build odd cycles with lengths in the range from $(r+6)$ to $(r-2+2 s)$ (see Fig. $3(\mathrm{~b}))$. Then, by using the above technique on every two rows of $M_{r \times s}$, every odd cycle in the range from $(r+2)$ to $(r-1) s+1$ can be built (see Fig. 3 (c)). To include the vertices in the last row into the above $((r-1) s+1)$-cycle, we can construct disjoint 4-cycles in the last two rows one by one from the second column of $M_{r \times s}$. Then use the combining operator "o" to combine the newly created cycle and a 4-cycle for obtaining a larger odd cycle (see Figs. 3 (d) and 3 (e) for $n$ is even and odd, respectively). This establishes the lemma.

Lemma 8: If $\operatorname{GR}\left(h_{k}, h_{k-1}, \ldots, h_{1}\right)$ has $h_{1}=\delta-1$, then it contains odd cycles of every length in the range from $\delta$ to $n$.

Proof. By Lemma 3 again, a mesh $M_{r \times s}$ with $r=\prod_{i=2}^{k} h_{i}$ and $s=h_{1}$ can be embedded in GR. By Lemma 6, $(0,0, \ldots, 0) \stackrel{1^{+h_{1} 2^{-}}}{\longrightarrow}(0,0, \ldots, 0)$ is a $\delta$-cycle (see Fig. $4(\mathrm{a})$ ). To construct cycles of lengths in the range from $\delta+2$ to $\delta+2(r-1)$, we can use the vertices in the second and third columns of $M_{r \times s}$ to build adjacent $C_{4}$ 's one by one from the first row to the last row of $M_{r \times s}$. Note that the bottom edge of a 4-cycle will be the top edge of the successive 4-cycle. Then use the combining operator "o" to combine 


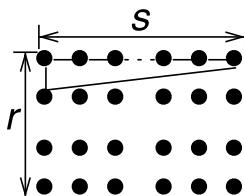

(a)

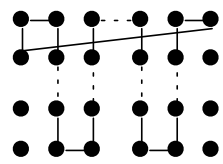

(d)

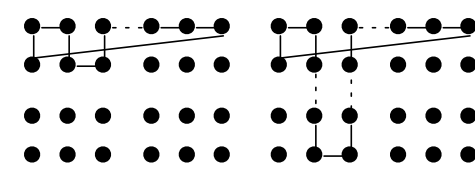

(b)

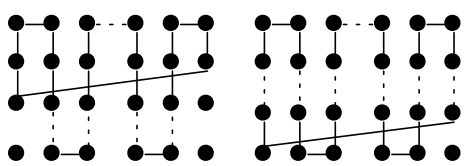

(e)

(f)
Fig. 4 Illustrations for Lemma 8 .

the newly created cycle and a $C_{4}$ for getting a larger odd cycle (see Figs. 4(b) and 4(c)). By using a similar technique on every two columns, i.e., columns 4 and 5, 6 and 7, etc., we can construct odd cycles with lengths in the range from $\delta+2 r$ to $\delta+(s-2)(r-1)$ (see Fig. 4(d)). In the above construction, we can find that only two vertices in the first column and one vertex in the last column, namely vertices $(0,0, \ldots, 0),(0,0, \ldots, 1,0)$, and $\left(0,0, \ldots, h_{1}-1\right)$, are used in constructing aforementioned odd cycles. By combining the previous constructed $C_{\delta+(s-2)(r-1)}$ and the $C_{4}=$ $(0,0, \ldots, 1,0) \stackrel{1^{-} 2^{+} 1^{+} 2^{-}}{\longrightarrow}(0,0, \ldots, 1,0)$, an odd cycle of length $\delta+(s-2)(r-1)+2$ is built (see Fig. 4 (e)). Similarly, by shifting down the above $C_{4}$ one row, another $C_{4}$ can be constructed, and, then combining with the newly created cycle, a larger odd cycle is built. By repeating the above procedure until the vertex in the lower left corner, namely vertex $\left(h_{k}-1, h_{k-1}-1, \ldots, h_{2}-1,0\right)$, is included, we have constructed all odd cycles for every length in the range from $\delta$ to $r \cdot s-1$ (see Fig. 4 (f)). This completes the proof.

Lemma 9: If $\mathrm{GR}\left(h_{k}, h_{k-1}, \ldots, h_{1}\right)$ is not a bipartite graph, then it contains odd cycles of every length in the range from $\delta$ to $n$.

Proof. Since GR is not bipartite, by Lemma 2, either $h_{k}$ is odd or $h_{i}$ is even for some $i \in\{1,2, \ldots, k-1\}$. By Lemmas 7 and 8 , if $h_{k}=\delta$ or $h_{1}=\delta-1$, then this lemma holds. It remains to consider the case that $h_{i}=\delta-1$ for some $1<i<k$. Let $\mathrm{GR}_{j}\left(h_{k}, h_{k-1}, \ldots, h_{i}\right)$ (GR $\mathrm{GR}_{j}$ for short) be the $j$ th copy of $\operatorname{GR}\left(h_{k}, h_{k-1}, \ldots, h_{i}\right)$, and, by definition, there are $\prod_{x=1}^{i-1} h_{x}$ such copies. By Lemma 3, there exists a mesh $M\left(=M_{r \times s}\right)$ with $r=\prod_{x=i}^{k} h_{x}$ and $s=\prod_{x=1}^{i-1} h_{x}$ such that $\mathrm{GR}_{j}$ is embedded in the $j$ th column of $M$. Note that the vertices in each column of $M$ are arranged according to their corresponding ordering in the largest odd cycle, say $C_{\ell}$, in $\mathrm{GR}_{1}$. We use $M^{\prime}\left(=M_{r \times s^{\prime}}\right)$ to denote the submesh of $M$ which is the mesh without containing the first column of $M$ (i.e., $\left.s^{\prime}=s-1\right)$. Also, we denote $M(p, q)$ the vertex at the $p$ th row and the $q$ th column of $M$. By Lemma 8, there exist odd cycles of every length in the range from $\delta$ to $r$ in $\mathrm{GR}_{1}$. All we have to prove is that there exist odd cycles of every length in the range from $\ell+2$ to $n$. Since $r$ is an even number, $C_{\ell}=C_{r}-1$. We consider the following cases.
Case 1. $s>2$. In this case, $M^{\prime}$ is still a mesh having more than one column. Since $M^{\prime}$ is bipancyclic, it contains every cycle of even length in the range from 4 to $r \cdot s^{\prime}$. It is obvious that we can construct every even-length cycle in $M^{\prime}$ such that there is an edge in the first column of $M^{\prime}$. Let $C_{x}$ be such an even cycle with an edge in the first column of $M^{\prime}$ which connects vertices $M^{\prime}(y, 1)$ and $M^{\prime}(y+1,1)$, namely $M(y, 2)$ and $M(y+1,2)$, respectively. Let $C_{4}=(M(y, 1), M(y, 2), M(y+1,2), M(y+1,1), M(y, 1))$. Since the cycle $C_{\ell}$ in $G R_{1}$ has an edge between $M(y, 1)$ and $M(y+1,1)$ for some $y$, we can find that $\left(C_{\ell} \circ C_{4}\right) \circ C_{x}$ is an odd cycle of length $\ell+x$. Note that $C_{\ell} \circ C_{4}$ is an odd cycle of length $\ell+2$. Therefore, in this case, there exists every odd cycle with length in the range from $\delta$ to $n-1$.

Case 2. $s=2$. In this case, there is only one column in $M^{\prime}$. However, there exists every even-length cycle, say $C_{x}$ for $4 \leqslant x \leqslant r$, in $\mathrm{GR}_{2}$ passing through the edge between vertices $M(1,2)$ and $M(2,2)$. Let $C_{4}=$ $(M(1,1), M(1,2), M(2,2), M(2,1), M(1,1))$. Thus, $C_{\ell} \circ C_{4}$ is an odd cycle of length $\ell+2$, and $\left(C_{\ell} \circ C_{4}\right) \circ C_{x}$ is an odd cycle of length $\ell+x$. This establishes the lemma.

Lemma 10: If $\mathrm{GR}\left(h_{k}, h_{k-1}, \ldots, h_{1}\right)$ is not a bipartite graph, then no odd cycle has length smaller than $\delta$.

Proof. If $k=1$, then there is exactly one cycle $C_{h_{1}}$ in GR, and this lemma holds directly. Therefore, we consider the case where $k \geqslant 2$ in the following. Suppose to the contrary that there is an odd cycle $C_{\ell}$ in GR with $\ell<\delta$ and no smaller odd cycle (including $C_{\ell}$ ) is totally contained in some $\mathrm{GR}_{i}\left(h_{k}, h_{k-1}, \ldots, h_{2}\right)$ for $1 \leqslant i \leqslant h_{1}$; otherwise, we can consider the subgraph $\mathrm{GR}_{i}$. Assume that $C_{\ell}=x \stackrel{j_{1} j_{2} \cdots j_{\ell}}{\longrightarrow} x$ where $j_{i}$ is a jump for $i=1,2 \ldots, \ell$. By Proposition 5 , we can swap jumps in $j_{1}, j_{2}, \ldots, j_{\ell}$ such that all jumps $1^{+}$are in the beginning, then follow by all jumps $1^{-}$, finally all other jumps appear without changing their order. For simplicity, we assume that the resulting jump sequence is $1^{+t_{1}}, 1^{-t_{2}}, j_{t_{1}+t_{2}+1}, \ldots, j_{\ell}$ for some nonnegative integers $t_{1}$ and $t_{2}$ and $j_{i} \notin\left\{1^{+}, 1^{-}\right\}$for $t_{1}+t_{2}+1 \leqslant i \leqslant \ell$. Clearly,

$$
x \stackrel{1^{+t_{1}} 1^{-t_{2}} j_{t_{1}+t_{2}+1} \cdots j_{\ell}}{\longrightarrow} x .
$$

In the following, we only consider the case where $t_{1} \geqslant t_{2}$. The case where $t_{1} \leqslant t_{2}$ can be handled similarly. By the possible values of $t_{2}$, there are two cases to be considered. Case 1. $t_{2}=0$. In this case, $t_{1}$ cannot be equal to 0 either; for otherwise, $C_{\ell}$ will be totally contained in some $\mathrm{GR}_{i}$ which is a contradiction. Therefore, $t_{1}$ must be equal to $h_{1}$. If $h_{1}$ is even, then $x \stackrel{1^{+h_{1} 2^{-}}}{\longrightarrow} x$ will be an odd cycle of length $h_{1}+1 \leqslant \ell$. However, by the definition of $C_{\delta}$ and the above inequality, $\delta \leqslant h_{1}+1 \leqslant \ell$. This contradicts the assumption that $\ell<\delta$. Therefore, $h_{1}$ must be an odd number. However, in this case, by replacing $1^{+t_{1}}$ with $2^{-}$in the jump sequence $1^{+t_{1}}, j_{t_{1}+1}, \ldots, j_{\ell}$, this results in a path $P=x \stackrel{2^{-} j_{t_{1}+1} \cdots j_{\ell}}{\longrightarrow} x$ which is totally contained in some $\mathrm{GR}_{i}$. Note that the length of $P$ is equal to $\ell-t_{1}+1$ which is less than $\ell$ and is an odd number. If every vertex in $P$ appears at most once, then $P$ 
is actually an odd cycle which contradicts the assumption. If some vertex in $P$ appears more than once, then a smaller odd cycle can be found in $P$. This is also a contradiction. Therefore, this case is impossible.

Case 2. $t_{2} \neq 0$. Obviously, if $t_{2} \neq 0$, then $x$ taking jumps $1^{+t_{2}} 1^{-t_{2}}$ will reach itself. This means that $x$ taking the remaining jump sequence $1^{+\left(t_{1}-t_{2}\right)}, j_{t_{1}+t_{2}+1}, \ldots, j_{\ell}$ still can reach itself, namely

$$
x \stackrel{1^{+\left(t_{1}-t_{2}\right)} j_{t_{1}+t_{2}+1} \cdots j_{\ell}}{\longrightarrow} x .
$$

Note that $\ell-2 t_{2}$ is still an odd number. Then, by a similar argument as Case 1, this case is also impossible. This completes the proof.

By combining Lemma 4 and Lemmas 7 10, we summarize our results as the following theorem.

Theorem 11: $\operatorname{GR}\left(h_{k}, h_{k-1}, \ldots, h_{1}\right)$ is $\delta$-pancyclic if $\delta$ exists, where $\delta$ is the minimum odd number in the set $\left\{h_{k}, h_{k-1}+1, h_{k-2}+1, \ldots, h_{1}+1\right\}$.

Corollary 12: A generalized recursive circulant graph $\operatorname{GR}\left(h_{k}, h_{k-1}, \ldots, h_{1}\right)$ is pancyclic if and only if $\delta=3$.

\section{Conclusion Remarks}

In this paper, we study the cycle embedding in a class of circulant graphs, which is a generalization of the class of RCGs. At first, we prove that a $k$-GRCG with two or more dimensions must be bipancyclic. Further, if we can determine the smallest odd $s$-cycle in a $k$-GRCG, then the graph also contains all odd cycles with length greater than $s$.

With a structure similar to multidimensional torus networks [6], the topology of the $k$-GRCGs provides an alternative for designing the parallel computer. For a future research, many network properties, as well as combinatorial problems can be studied on $k$-GRCGs.

\section{Acknowledgments}

This research was partially supported by MOST grants 104-2221-E-011-023-MY3 (Yue-Li Wang) and 104-2221E-141-002-MY3 (Jou-Ming Chang), from the Ministry of Science and Technology, Taiwan.

\section{References}

[1] S.B. Akers and B. Krishnamurthy, "A Group-theoretic model for symmetric interconnection networks," IEEE Tran. Comput., vol.38, no.4, pp.555-566, 1989.

[2] T. Araki, "Edge-pancyclicity of recursive circulants," Inf. Process. Lett., vol.88, no.6, pp.287-292, 2003.

[3] T. Araki and Y. Shibata, "Pancyclicity of recursive circulant graphs," Inf. Process. Lett., vol.81, no.4, pp.187-190, 2002. Erratum, vol.84, no.3, p.173, 2002.

[4] N. Biggs, Algebraic Graph Theory, 2nd edition, Cambridge University Press, 1993.

[5] D.K. Biss, "Hamiltonian decomposition of recursive circulant graphs," Discrete Math., vol.214, no.1-3, pp.89-99, 2000.
[6] L.N. Bhuyan, and D.P. Agrawal, "Generalized hypercube and hyperbus structures for a computer network," IEEE Tran. Comput., vol.33, no.4, pp.323-333, 1984

[7] F.T. Boesch and R. Tindell, "Circulants and their connectivity," J. Graph Theory, vol.8, no.4, pp.487-499, 1984.

[8] F. Buckley and F. Harary, Distance in Graphs, Addison-Wesley, Redwood City, CA, 1990.

[9] Y.-C. Chen and T.-H. Tsai, "Hamiltonian decomposition of generalized recursive circulant graphs," Inf. Process. Lett., vol.116, no.9, pp.585-589, 2016.

[10] Y.-C. Chen, L.-H. Hsu, and J.J.-M. Tan, "A recursively construction scheme for super fault-tolerant hamiltonian graphs," Appl. Math. Comput., vol.177, no.2, pp.465-481, 2006.

[11] D. Choi and I. Chung, "Application of the Hamiltonian circuit Latin square to a parallel routing algorithm on generalized recursive circulant networks," J. Korea Multimedia Society, vol.18, no.9, pp.1083-1090, 2015.

[12] I. Chung, "Construction of a parallel and shortest routing algorithm on recursive circulant networks," Proc. 4th International Conference on High Performance Computing in the Asia-Pacific Region, Beijing, China, pp.580-585, 2000.

[13] L.-H. Hsu and C.-K. Lin, Graph Theory and Interconnection Networks, CRC Press, Boca Raton, FL, 2008.

[14] C. Kim, J. Choi, and H.-S. Lim, "Embedding full ternary trees into recursive circulants," Proc. First EurAsian Conference on Information and Communication Technology, Shiraz, Iran, pp.874-882, 2002.

[15] S. Kim and I. Chung, "Application of the special Latin square to a parallel routing algorithm on a recursive circulant network," Inf. Process. Lett., vol.66, no.3, pp.141-147, 1998.

[16] S.-Y. Kim, J.-H. Lee, and J.-H. Park, "Disjoint path covers in recursive circulants $G\left(2^{m}, 4\right)$ with faulty elements," Theoret. Comput. Sci., vol.412, no.35, pp.4636-4649, 2011.

[17] S.-Y. Kim and J.-H. Park, "Paired many-to-many disjoint path covers in recursive circulants $G\left(2^{m}, 4\right)$," IEEE Tran. Comput., vol.62, no.12, pp.2468-2475, 2013.

[18] H-.S. Lim, J.-H. Park, and K.-Y. Chwa, "Embedding trees in recursive circulants," Discrete Appl. Math., vol.69, no.1-2, pp.83-99, 1996.

[19] S.A. Mane and B.N. Waphare, "Regular connected bipancyclic spanning subgraphs of hypercubes," Comput. Math. Appl., vol.62, no.9, pp.3551-3554, 2011.

[20] C. Micheneau, "Disjoint Hamiltonian cycles in recursive circulant graphs," Inf. Process. Lett., vol.61, no.5, pp.259-264, 1997.

[21] J.-H. Park, "Panconnectivity and edge-pancyclicity of faulty recursive circulant $G\left(2^{m}, 4\right)$," Theoret. Comput. Sci., vol.390, no.1, pp.70-80, 2008.

[22] J.-H. Park and K.-Y. Chwa, "Recursive circulant: A new topology for multicomputer networks," Proc. International Symposium on Parallel Architectures, Algorithms and Networks, Kanazawa, Japan, pp.73-80, 1994.

[23] J.-H. Park and K.-Y. Chwa, "Recursive circulants and their embeddings among hypercubes," Theor. Comput. Sci., vol.244, no.1-2, pp.35-62, 2000.

[24] I. Stojmenović, "Multiplicative circulant networks topological properties and communication algorithms," Discrete Appl. Math., vol.77, no.3, pp.281-305, 1997.

[25] S.-M. Tang, Y.-L. Wang, and C.-Y. Li, "Generalized Recursive Circulant Graphs,” IEEE Tran. Comput., vol.23, no.1, pp.87-93, 2012.

[26] C.-H. Tsai, J.J.M. Tan, Y.-C. Chuang, and L.-H. Hsu, "Hamiltonian properties of faulty recursive circulant graphs," J. Interconnection Networks, vol.3, no.3-4, pp.273-289, 2002.

[27] C.-H. Tsai, J.J.M. Tan, and L.-H. Hsu, "The super-connected property of recursive circulant graphs," Inf. Process. Lett., vo.91, no.6, pp.293-298, 2004.

[28] J.-M. Xu, Topological Structure and Analysis of Interconnection Networks, Kluwer Academic Publishers, Dordrecht/Boston/London, 
2001.

[29] J.-M. Xu, M. Ma, "Survey on cycle and path embedding in some networks," Front. Math. China, vol.4, no.2, pp.217-252, 2009.

[30] J.-S. Yang, J.-M. Chang, S.-M. Tang, and Y.-L. Wang, "On the independent spanning trees of recursive circulant graphs $G\left(c d^{m}, d\right)$ with $d>2$," Theor. Comput. Sci., vol.410, no.21-23, pp.2001-2010, 2009.

[31] J.-S. Yang, J.-M. Chang, S.-M. Tang, and Y.-L. Wang, "Constructing multiple independent spanning trees on recursive circulant graphs $G\left(2^{m}, 2\right)$, , Int. J. Found. Comput. Sci., vol.21, no.1, pp.73-90, 2010.

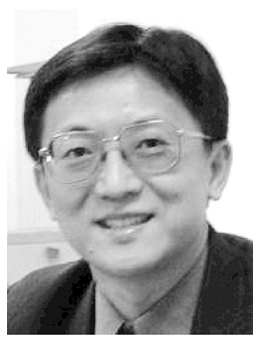

Shyue-Ming Tang received his M.S. degree in computer and information science (1992) from Temple University (Philadelphia, USA). In 2002, he received his Ph.D. degree in information management from the National Taiwan University of Science and Technology. He is a professor in National Defense University now. His research interests include interconnection networks, graph theory and algorithm design.

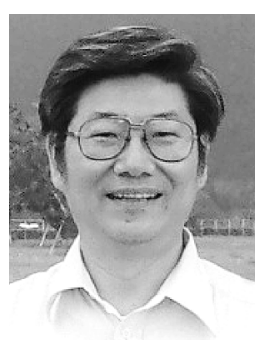

Yue-Li Wang received the B.S. and M.S. degrees both in Information Engineering Department of Tam-Kang University in 1975 and 1979, respectively. In 1988, he received the Ph.D. degree in information engineering from the National Tsing-Hua University. Now, he is a professor in the Department of Information Management of National Taiwan University of Science and Technology. His research interests include graph theory, algorithm analysis and parallel computing.

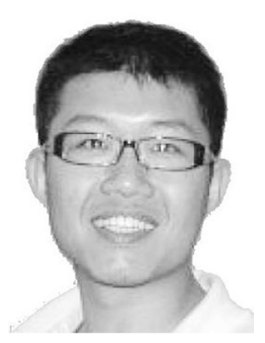

Chien-Yi Li received the M.S. degree in Information Management Department of National Formosa University in 2008. He is now working toward the Ph.D. degree in Information Management Department of National Taiwan University of Science and Technology. His research interests include graph theory and algorithm.

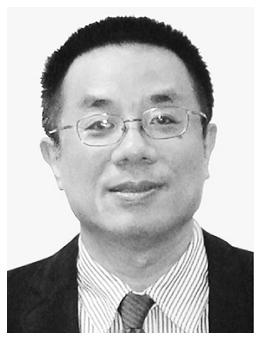

Jou-Ming Chang received his B.S. degree in applied mathematics from Chinese Culture University, Taipei, Taiwan, in 1987, the M.S. degree in information management from National Chiao Tung University, Hsinchu, Taiwan, in 1992, and the Ph.D. degree in computer science and information engineering from $\mathrm{Na}-$ tional Central University, Zhongli, Taiwan, in 2001. Currently, he is a distinguished professor in the Institute of Information and Decision Sciences at the National Taipei University of Business (NTUB). He has been served on the dean of the College of Management at NTUB in 2014. His major research interest includes algorithm design and analysis, graph theory, and parallel and distributed computing. 